\title{
The UAV Control Approach by using Multi Agent Systems
}

\author{
Adrian Muraru ${ }^{1}$, Ramona Lile ${ }^{2}$, Elena Corina Boșcoianu ${ }^{3}$, Mircea Boșcoianu ${ }^{1}$, Luigie Vlădreanu ${ }^{4}$ \\ ${ }^{1}$ Departement of engineering and industrial management, Transilvania University of Brașov \\ ${ }^{2}$ Aurel Vlaicu University of Arad \\ ${ }^{3}$ Department of Structures and Materials, National Institute for Aerospace Research "Elie Carafoli", Bucharest \\ ${ }^{4}$ Department of Mechatronics, Institute of Solid Mechanics of the Romanian Academy, Romanian Academy, Bucharest
}

\section{Article Info}

Received Jan $12^{\text {th }}, 2019$

\section{Keyword:}

multi agent system unmanned aerial vehicles air traffic management autonomous control systems negotiating flight conflicts

\begin{abstract}
The Unmanned Aerial Vehicles (UAV) are becoming more and more accessible and their applications will grow with various utility's from military fields with specific missions to civil so that in-depth research, in which all this types of UAV's will operate in the future airspace, has to be done via a new concept on systems centralized autonomous control system. Approach of a Multi-Agent System (MAS) used for the development of an autonomous UAV Control System is presented. In the proposed model some processes are simplified, however it is unique, and can be used as it to help UAV's operate in the flight airspace. This system will serve many UAV users to help them work in the same defined airspace without concerns regarding safety. The proposed model is a new concept of a multi-agent system design, simulated in NetLogo software, which include the parameters necessary to function with the objective to maintaining separation.
\end{abstract}

\section{Corresponding Author:}

Luige Vladareanu,

Romanian Academy,

Institute of Solid Mechanics of the Romanian Academy,

15 Constantin Mille street, 010141 Bucharest, Romania.

Email: luige.vladareanu@vipro.edu.ro

\section{Introduction}

The term Unmanned Aerial Vehicles (UAV) is defined as a type of non-powered aircraft that does not carry a human pilot, uses aerodynamic forces to prove ground clearance, can fly autonomously or be remotely controlled, can be recoverable or consumable, and can carry a lethal or non-lethal payload [1]. The demand for UAVs has increased significantly in recent years. These are preferred for pilots because they have: low costs; multiple mission capabilities; simplicity; ability to perform dirty and dangerous missions that cannot be done by piloted aircraft's.

UAVs are used in military applications for observation missions such as surveillance, reconnaissance, air support and monitoring for many years. Their adaptation to civilian missions has become more serious in recent years. Some variants of civil applications are search and rescue, disaster monitoring, meteorological data acquisition, and maritime monitoring [2].

Weather factors are effects of environmental conditions on the aircraft and the track. Technical team and decision-makers are the errors of pilots and crew. System Factor are failures of the aircraft subsystems. Automatic landing at an UAV increases overall system autonomy and adds consistency. The automatic pilot landing ensures greater safety and reduces the load of pilots and crew in operations. It also increases winds at aircraft and allows landing in situations with strong winds, turbulence and shear forces. 
Creating a dynamic model of an aircraft is an important milestone in the design of the automatic pilot. Dynamic models include detailed information about aerodynamics-based system features, propulsion system, mass-inertia properties and drive dynamics. The inertia of the aircraft is calculated by experiments, simulations built using such methods offer low costs and efficient time solutions for small UAVs. They average long-term static aerodynamic aerodynamics which are obtained using the Dynamics of Computing Fluids (DCF), while dynamic derivatives are discovered using semi-empirical formulas. DCF results are more reliable than empirical methods, but getting them takes longer time. In the work of Ippolito [3], the dynamic characteristics of the system are obtained by flight tests.

In modern control theory, some methods such as adaptive control, robust control, fuzzy logic, are introduced due to uncertainties caused by non-linearity [4], [5], [6]. The transition from the forward flight to the planning of a fixed UAV wing is accomplished by Johnson [7] by adding a neural network of online learning to an inversion model based on a classic operator to take into account uncertainties. Although UAV dynamics is very different in planning and forward flight, neural networks are able to compensate for the difference in the above study.

\section{Using Multi Agents System in modeling}

The term agent has been used with several meanings, from a command line to an adaptive, intelligent, autonomous entity [8], in this paper we will use Srinivasan \& Jain [9] definition as an interactive and autonomous entity. From a historical point of view, the creation of agents has the goal on modeling intelligent agents to achieve a degree of autonomy.

In recent years, the researchers, has applied simulations of several agents that can interact with one another. Multi-agent systems (MAS) have two requirements: building autonomous entities with interactive agents and testing how the system behaves as a result of common actions and interference between agents. The simulations are carried out on a large scale of social sectors such as telecommunications, industrial and military.

The model proposed here is concentrated on specific aspects of the system regarding its behavior needs on one hand and on the other hand in forming an agent based simulation. Exact prediction of the behavior at a high level is necessary to model aspect at the very low level of its structure. Additionally, taking into account our unique accent on air traffic models, we can assess that a rich environment which defines the physical aspects of the agent behavior is needed to help in establishing the inner-mechanism between agents [10].

Another model of MAS has concentrated on closed systems, in which most aspects of agents can be determined [11]. Closed systems have a number of uses, such as teamwork, dedicated applications and distributed sensor networks where the role of agents is to standardize with the goals of the system. These simulation uses models of complex and heterogeneous agents that can perform different roles and functions.

MAS with different capabilities and desires, beliefs, co-ordination and collaboration between them are needed. Coordination refers to the long-term management of events, tasks and actions between agents. Collaboration also needs agents to have the same goals and intentions [12].

Modelling used in cognitive engineering such as the Air Traffic Management ATM system shows us the following characteristics [13], this makes them desirable in agent-based simulations:

1. Involve in a variety of roles a number of agents with a variety of intentions;

2. Targeting precise goals or objectives;

3. Having pre-established knowledge, culture, and process;

4. Capable of affecting and being affected by the operating environment.

The first character can identify the air traffic management system as an open agent-based system. Agents work to simulate system requirements, using a heterogeneous set they assign their roles and variables that are precisely captured. The second characteristic of the air traffic management system shows us that their programmers want to mimic "micro-level" behavior in order to achieve some higher-level "macro-level" goals, as specified in the procedures for air traffic controllers and pilots that will make good air traffic flow patterns. In particular, methods for simulating focus on macro and micro behaviors simultaneously.

The third feature reflects the air traffic simulation configurations that need to be introduced into the models underlying the simulation. In the case of air traffic simulation, the agents and the environment are presented in simulation using the same models as the role of agents that is characterized by: finding agents and their environment, and exposing roles, procedures, tasks and capabilities. This type of representation need a level of effort proportional to implementation in real systems. 
The last feature copies its recent focus to viewing the agents' environment. The emphasis on an environmental model facilitates richer models of agents for whom cognitive status, cognitive distribution and expert environmental adjustment can be explicitly described. In the organizational concept many attributes of the environment are created as being divided between agents. The agent-based simulation shows regulatory, organizational environmental elements that offers a fabric in which agents can be implemented to help with the system's complete behavior.

\section{Design and simulation using Netlogo}

Version 6.0.2 of NetLogo is a programming language for agent-based creation and integration into a virtual environment it helps create emerging behavior and comes with a pre-existing database that helps modelling in various fields such as economics, biology, physics, chemistry, psychology, dynamic systems. It allows the exploration of patterns through modifiers such as buttons, levers, imputations, and other interface elements. Beyond exporting, NetLogo allows creating new models and modifying existing ones. NetLogo is an open source software that can be downloaded for free. It is used in a multitude of educational contexts [14].

Defining input data for the simulator, with the emergence and deployment of the Extended Mode (EHS) transponder and Automatic Dependent Surveillance - Broadcast (ADB-S) transponder, facilitates a wide range of data to be transmitted from the UAV to the ground, as part of the input data having access to each of the UAV's autopilot is required.

NetLogo is an open source software that can be downloaded for free. It is used in a multitude of educational contexts. The simulation is set inside the project with the following parameters: area: $50 \times 50 \mathrm{~km}$, altitudes: 1000 feet up 28000 feet. UAV's speed: randomly generate between $50 \mathrm{~km} / \mathrm{h}$ and $500 \mathrm{~km} / \mathrm{h}$. UAV's origin: randomly generate at the edge of the simulation or randomly generated at the specific points simulating UAV departure. UAV's destination: randomly generate at the edge of the simulation or randomly generated at the specific points simulating UAV arrivals. Factors: a restricted airspace in the S-E seen in fig. 1.

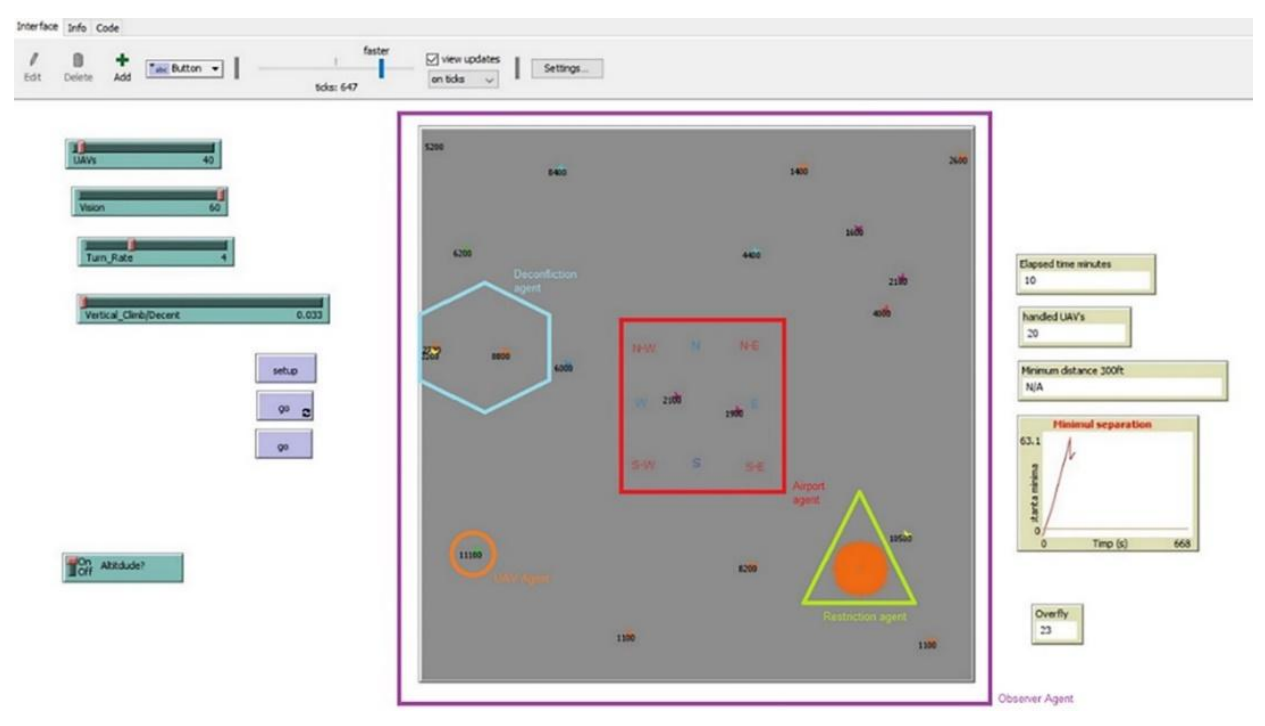

Figure 1: MAS main console Netlogo

The simulation has specific parameters that can be adjusted according to the user's desire:

a. UAV's = maximum number of UAV's in the simulation;

b. Vision $=$ a rage between 1 and $12 \mathrm{~km}$;

c. Turn rate: a parameter that lets all UAV's turn in specific degree/second between 1 - 9;

d. Vertical Climb / Descent = a parameter that lets all UAVțs set there climb/descent speed in thousands of feet per minute default is 2000 feet/minute.

The project has 5 types of agents: Observer, UAV's, Restriction, Deconflition and Airport see figure 1. 


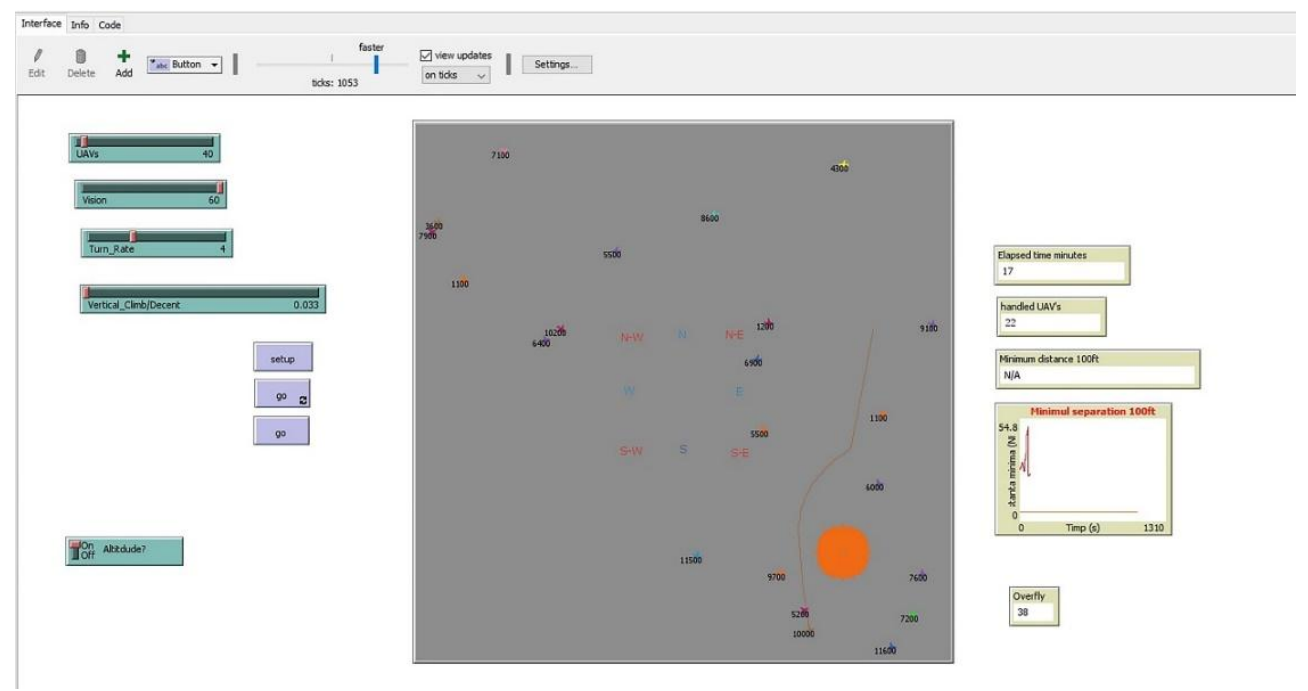

Figure 2: UAV's avoiding Restricted Airspace

For our simulation we are using a various number of UAV's from 40 to 70 with increments of 10 with the following parameters for the agent named UAV's: degree of roll: 4 degrees, vision range: $12 \mathrm{~km}$, vertical speed: $2000 \mathrm{feet} / \mathrm{minutes}$.

The combination of these parameters results in 4 simulation with 6 hours simulation runtime each, we will use as an indicator the maintaining of the minimum separation between UAV's horizontally $1 \mathrm{~km}$ and vertically $300 \mathrm{ft}$ with the ability of avoiding restricted airspace as seen in figure 2, with the ability to avoid each other as seen in figure 3 all the while the UAV's are fulfilling their individual objectives, respective to reach their desire destination.

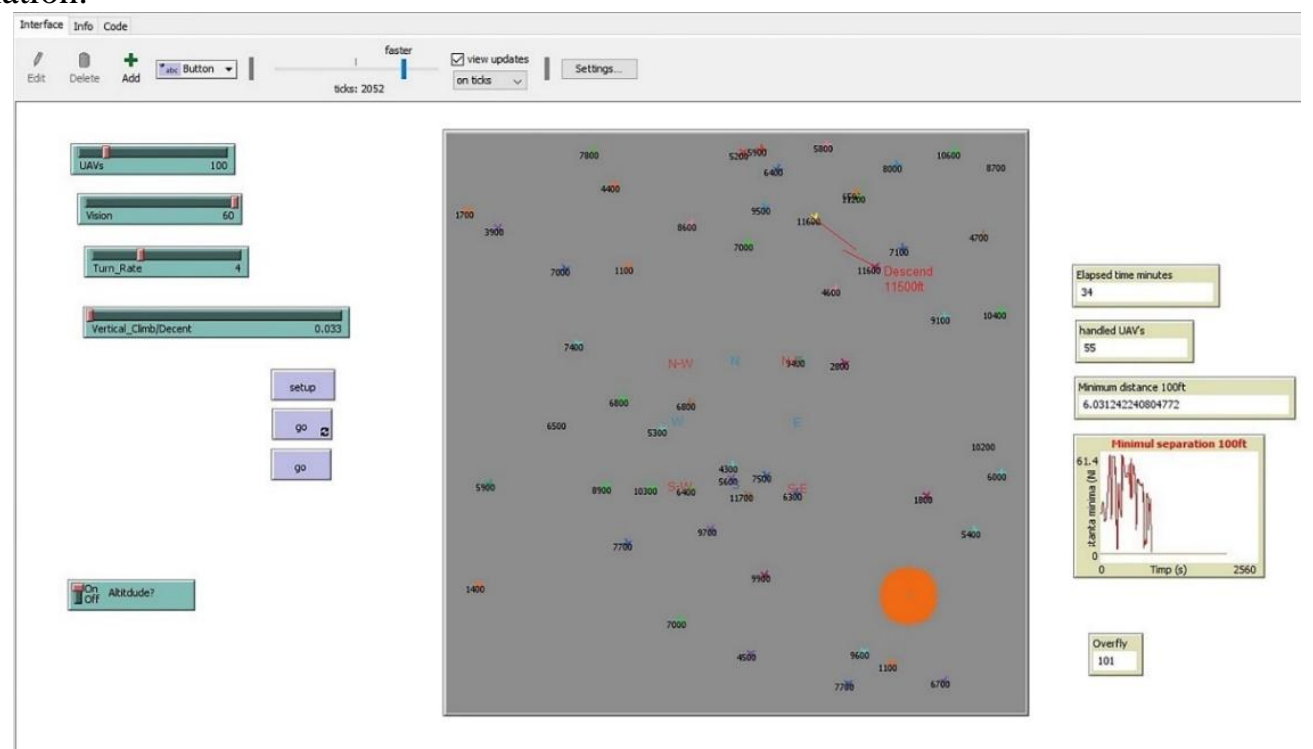

Figure 3. UAV's negotiating flight conflicts

The NetLogo software has the ability to track and record in Microsoft excel format data regarding the position and action taken by each agent on an one-second interval period, as such the key indicator for the project is the minima of two UAV's regarding vertical separation $300 \mathrm{ft}$ and horizontal separation of $1 \mathrm{~km}$ while avoiding the restricted airspace. 


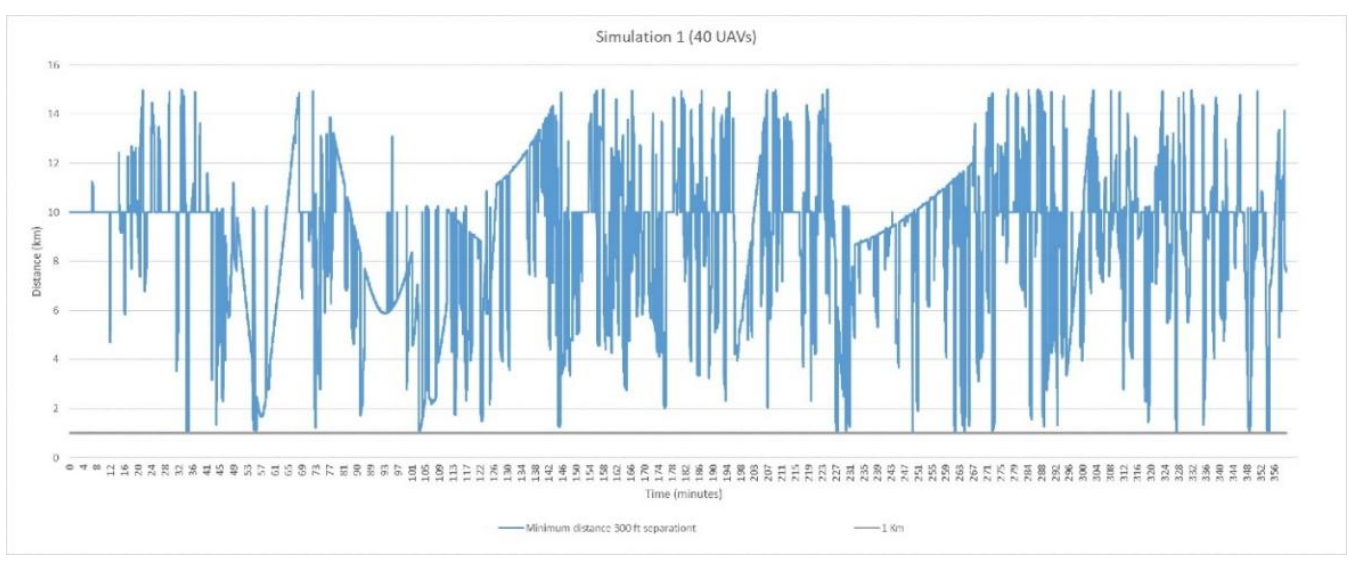

Figure 4. Simulation 140 UAV's

In the figures 4 we can see that throat the duration of the simulation 6 hours the blue line representing the closes minimum separation between 2 UAV's and the $1 \mathrm{~km}$ threshold is represented is grey colour.

Simulation 1 contains 40 UAV's as seen in the figure below the minimum distance between was 1.005697 $\mathrm{km}$, as the number of UAV's in the simulation grows so do the number of conflicts handled by the software. The simulation handled 692 UAV's in the 6 hour period with a peak of 53 UAV's at once.

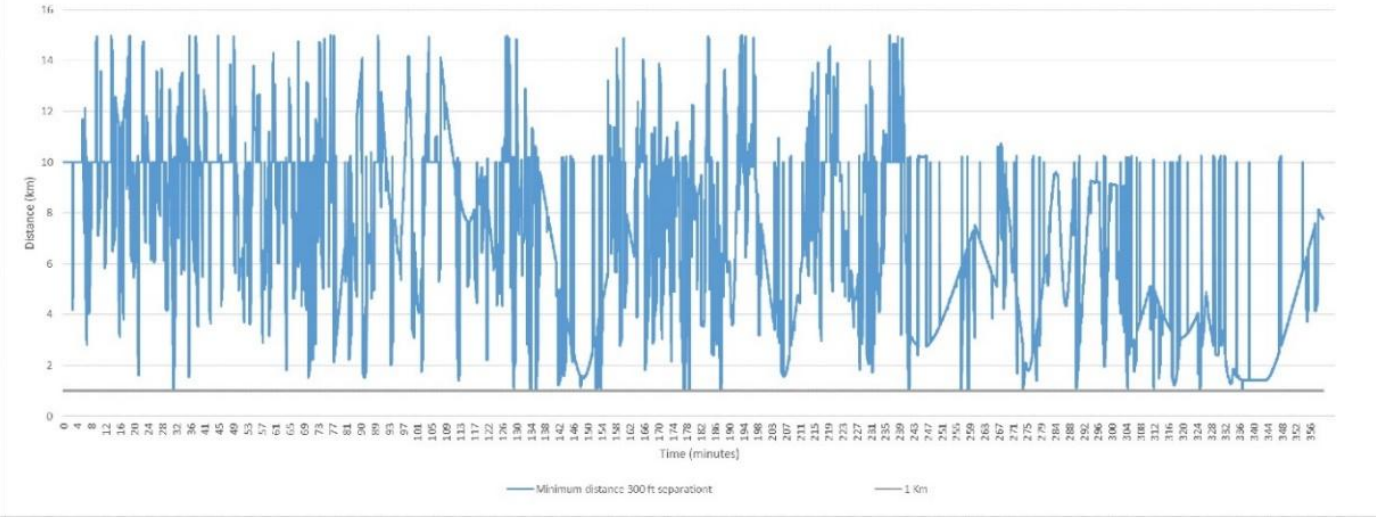

Figure 5: Simulation 250 UAV's

Seen in figure 4 simulation 2 contains 50 UAV's as seen in the figure below the minimum distance between was $1.000559621 \mathrm{~km}$, the simulation handled 868 UAV's in the 6-hour period with a peak of 62 UAV's at once.

Simulation 3 seen in figure 6, contains 60 UAV's as seen in the figure below the minimum distance between was $1.001324387 \mathrm{~km}$, the simulation handled 937 UAV's in the 6-hour period with a peak of 72 UAV's at once.

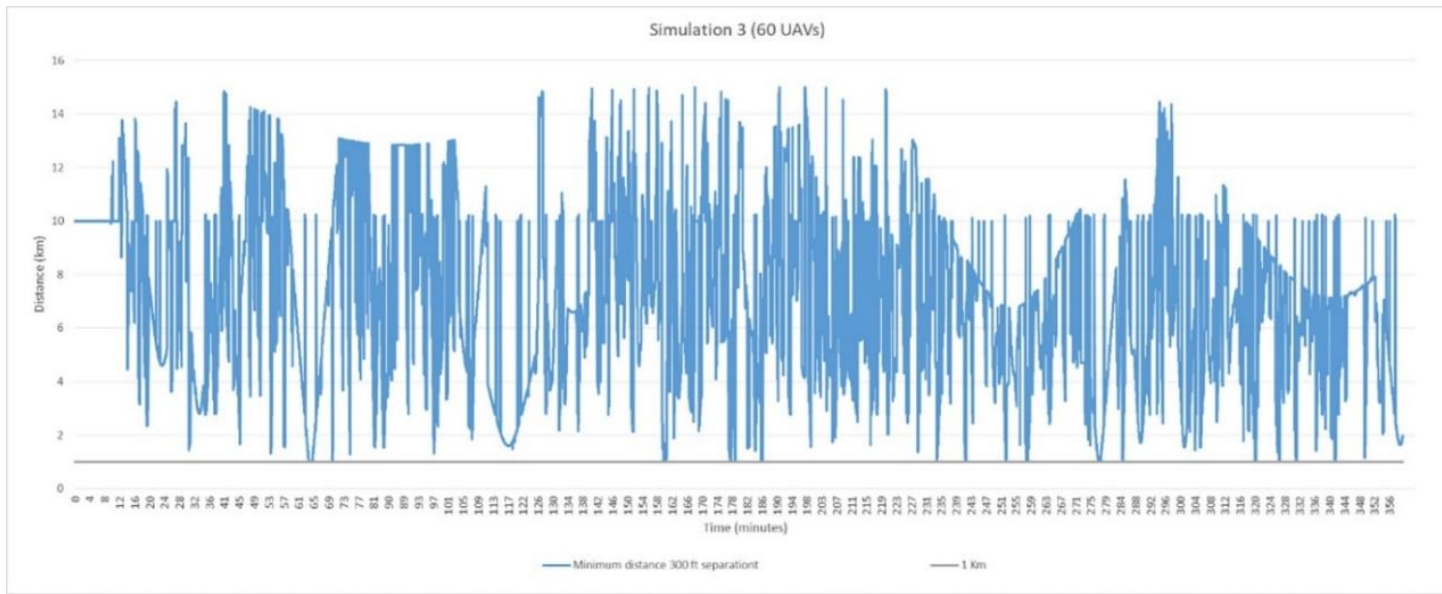

Figure 6: Simulation 360 UAV's 
In figure 7 named simulation 4 contains 60 UAV's as seen in the figure below the minimum distance between was $0.875086015 \mathrm{~km}$, as we can see the number is below our intended $1 \mathrm{~km}$ which indicates the model has reached its capacity limit, the simulation handled 1215 UAV's in the 6-hour period with a peak of 83 UAV's at once.

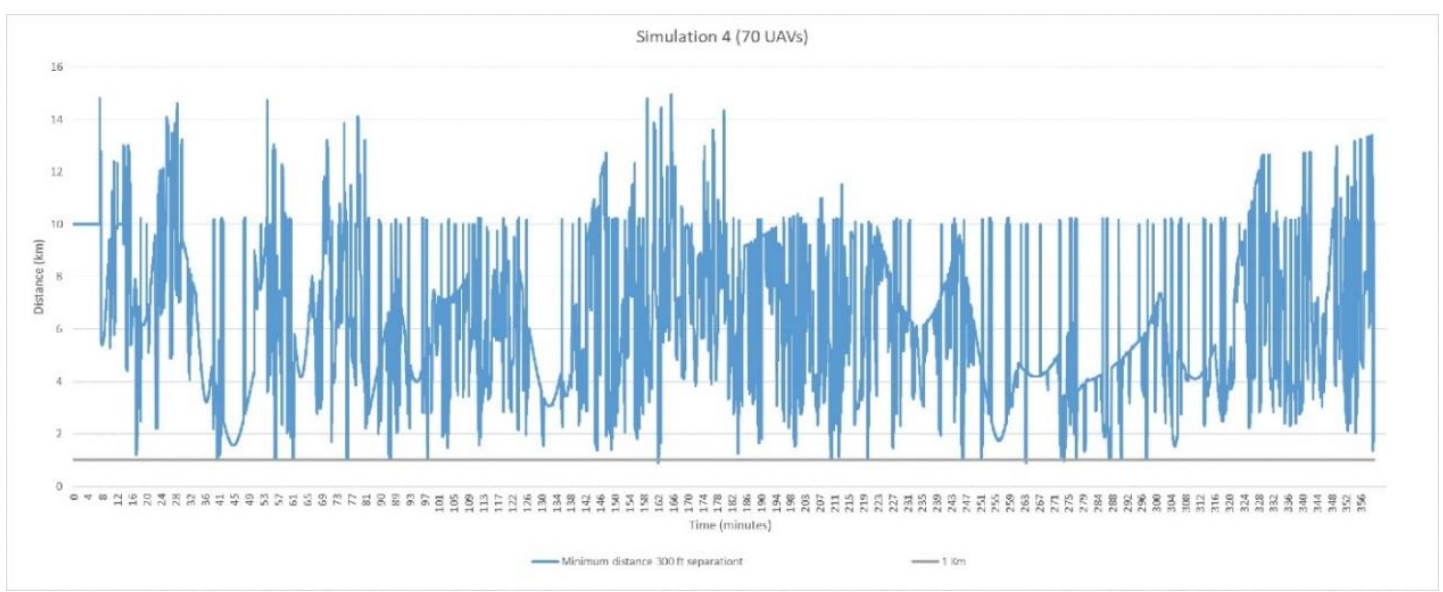

Figure 7: Simulation 470 UAV's

The system was designed to deconflict 2 or 3 UAV's at the same time as seen in the figure 3 , we can conclude that the model provides sufficient security to maintain 60 or fewer UAV's with simulated conditions in the area of responsibility over this number due to the complexity of the negotiation between agents is insufficient, resulting in conflicts of more than 3 UAV's requiring updates to the core MAS's design.

\section{Conclusions}

The proposed model is an UAV Control System based on the Multi-Agent System created in NetLogo that includes parameters required for operation within a modern airspace. This system will serve multiple UAV users.

The novelty of the paper is the actual design and validation of the UAV Control System this was done by simulating normal UAV operation in the airspace in the first part, and in the second part was the detection and the design of the deconfliction algorithm this was very important in proving the model.

In this model, some processes and details are simplified, and there are no similar models, it is believed that the use of the proposed model can be primarily used as safety tool. It will, in the future, provide the knowledge needed to describe and predict UAV congested areas under other conditions that cannot be taken into account at present.

\section{Acknowledgements}

This work was supported by a grant of the Romanian Ministry of Research and Innovation, CCCDIUEFISCDI, MULTIMOND2 project number PN-III-P1-1.2-PCCDI2017-0637/33PCCDI/01.03.2018, within PNCDI III, and by the European Commission Marie Skłodowska-Curie SMOOTH project, Smart Robots for Fire-Fighting, H2020-MSCA-RISE-2016-734875.

\section{References}

[1] Office of the Secretary of Defence. (2005). UAS Roadmap 2005-2030.

[2] Anastasiei, T., Muraru, A., \& Nencu, N. "Sense and Avoid Strategies for Modern Unmanned Aerial Systems." KBO- The 16th International Conference Sibiu: Academia Forţelor Terestre "Nicolae Bălcescu", pp. 358-363, 2010.

[3] Ippolito, C., Yoo-Hsui, Y., \& Kaneshige, J. "FSF Neural Adaptive Flight Control Testing on an Unmanned Experimental Aerial Vehicle." AIAA., 2007.

[4] Boșcoianu, M., Pahonie, R., \& Coman, A. "Some Aspects Regarding the Adaptive Control of a Flying Wing- Micro Air Vehicle with Flexible Wing Tips". WSEAS TRANSACTIONS on SYSTEMS. Bucharest: Department of Aviation and Technology Military Technical Academy, pp. 727-737, 2008 
[5] Vladareanu V., Dumitrache I., Vladareanu L, Sacală I. S., Tonţ G, Moisescu M.A., "Versatile Intelligent Portable Robot Control Platform Based on Cyber Physical Systems Principles", Studies in Informatics and Control, ISSN 1220-1766, vol. 24 (4), pp. 409-418.2015.

[6] Vladareanu L., Melinte O., Bruja A., Wang H.B., Wang X., Can S., "Haptic interfaces for the rescue walking robots motion in the disaster areas, Haptic interfaces for the rescue walking robots motion in the disaster areas", UKACC International Conference on Control (CONTROL) 2, UK, ISBN: 978-1-4799-5011-9, pp. 498 - 503, 2014 https://ieeexplore.ieee.org/document/6915190.

[7] Johnson, E., Turbe, M., Wu, A., Kannan, S., \& Neidhoefer, J. "Flight Test Results of Autonomous Fixed-Wing UAV Transitions to and from Stationary Hover". AIAA Guidance, Navigation and Control Conference. , 2006.

[8] D'Inverno, M., Luck, M., \& Luck, M. M. "Understanding Agent Systems.” Springer Science \& Business Media, 2004.

[9] Srinivasan, D., \& Jain, L. C. "Innovations în Multi-Agent Systems and Application - 1.” Springer Science \& Business Media, 2010.

[10] Hooshangi, N., \& Alesheikh, A. "Developing an Agent-Based Simulation System for PostEarthquake Operations in Uncertainty Conditions: A Proposed Method for Collaboration among Agents." ISPRS International Journal of Geo-Information, 2018 doi:https://doi.org/10.3390/ijgi7010027.

[11] Bisantz, A. M., \& Burns, C. M. "Applications of Cognitive Work Analysis." CRC Press, 2016.

[12] Lomuscio, A., Nepal, S., Patrizi, F., Benatallah, B., \& Brandić, I. "Service-Oriented Computing”-ICSOC 2013. Berlin: Springer International Publishig Switzerland, 2013.

[13] Muraru, A., \& Boșcoianu, M. „A Critical Analysis of the European Airspace Architecture.” Proceedings of the 5th Review of Management and Economic Engineering Engineering, International Management Conference, RMEE2016 - From Management of Crisis to Management in a Time of Crisis pp. 187-192, 2016 Cluj-Napoca: Todesco Publishing House.

[14] Wilensky, U. NetLogo, 6.0.2. (Center for Connected Learning and Computer-Based Modeling) Retrieved 1 15, 2018, from NetLogo: http://ccl.northwestern.edu/netlogo. 